Supplementary Information

\title{
Artificial Chameleon Skin with Super Sensitive Thermal and Mechanochromic Response
}

Yue Wu, Yu Wang, Shufen Zhang, and Suli Wu*

State Key Laboratory of Fine Chemicals, Dalian University of Technology, 2\# Linggong Road, Dalian 116024, P.R. China

Corresponding Author Email: wusuli@dlut.edu.cn 


\section{RESULTS AND DISCUSSION}

To confirm the property that the $\mathrm{ZnS}$ core cannot be etched by HF but the silica shell can, two kinds of PCs were prepared with $\mathrm{ZnS}$ and silica nanospheres as building bulks, respectively. Both PCs were immersed in HF solution for 30 minutes after being filled with polymers. The crosssection scanning electron microscope (SEM) images, and corresponding element distribution mapping images of the treated PCs film (Figure S1) reveals that the ZnS nanospheres still exist and silica nanospheres disappear. Comparing the reflection spectra of the $\mathrm{ZnS}$ PC films before and after etching (Figure S2), it can be found that the frequency and intensity of the reflection spectrum have not changed. The invariance of the spectrum means that the $\mathrm{ZnS}$ nanospheres were survived completely during the etching process without partially destroyed. Moreover, the reflectivity of the PC film with $\mathrm{ZnS} @ \mathrm{SiO}_{2}$ nanospheres as building bulks is stronger than that of the PC film composed of ZnS nanospheres (Figure S3).

Details of Bragg's law. In Equation $1, \lambda$ is the wavelength of reflection peak, $d$ is the diameter of the nanospheres, $\mathrm{m}$ is the Bragg reflection order, $n_{\mathrm{a}}$ is the effective refractive index, and $\theta$ is the angle between the normal and the plane of the crystals. In Equation 2, $n_{\mathrm{i}}$ is the refractive index of different components inside photonic crystals and $\varphi_{i}$ is the volume fraction of each $i$ portion. 

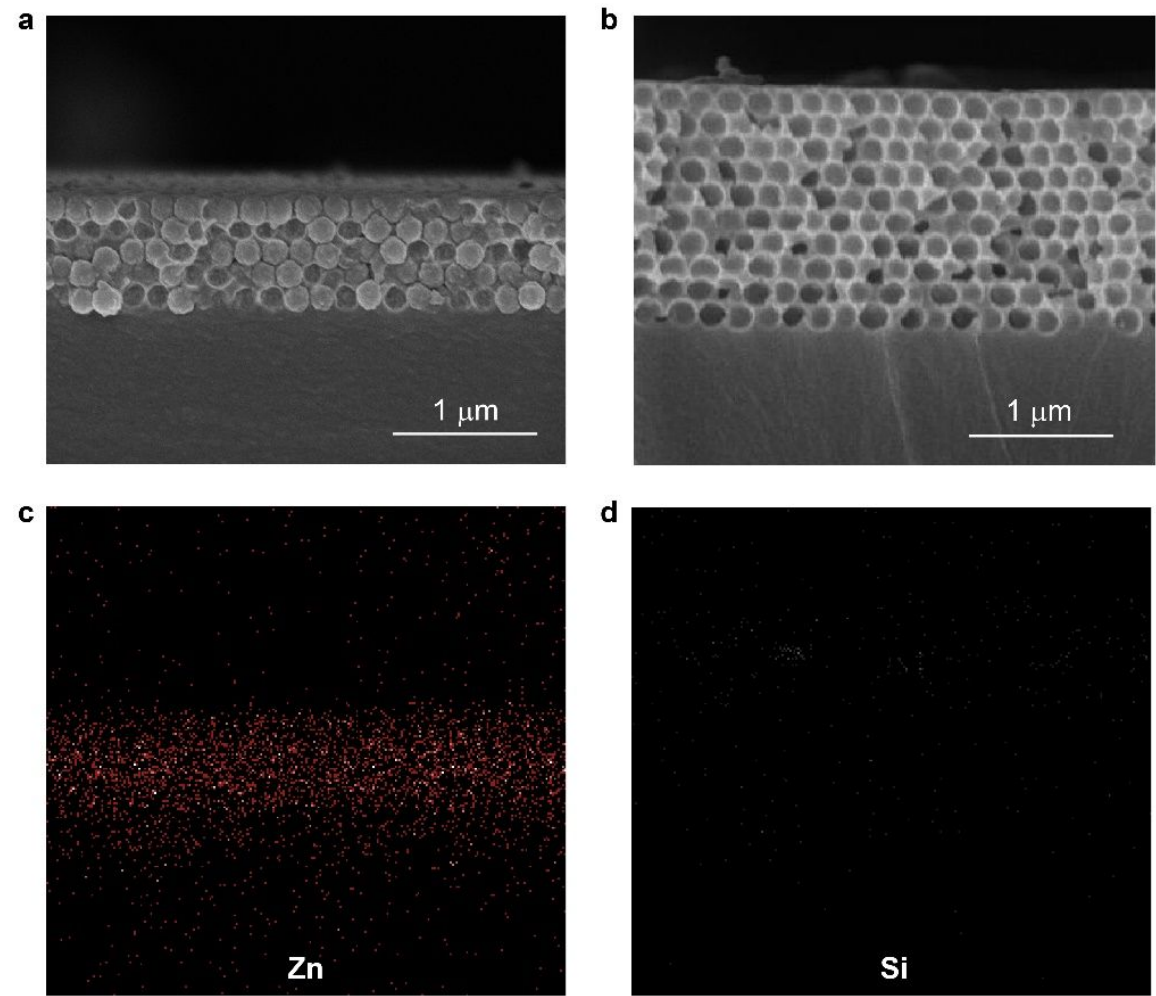

Figure S1. (a-d) SEM images and corresponding element maps of the PC films: (a, c) the PC film composed of $\mathrm{ZnS}$ nanospheres and polymer, $(\mathrm{b}, \mathrm{d})$ the $\mathrm{PC}$ film composed of $\mathrm{SiO}_{2}$ nanospheres and polymer. 


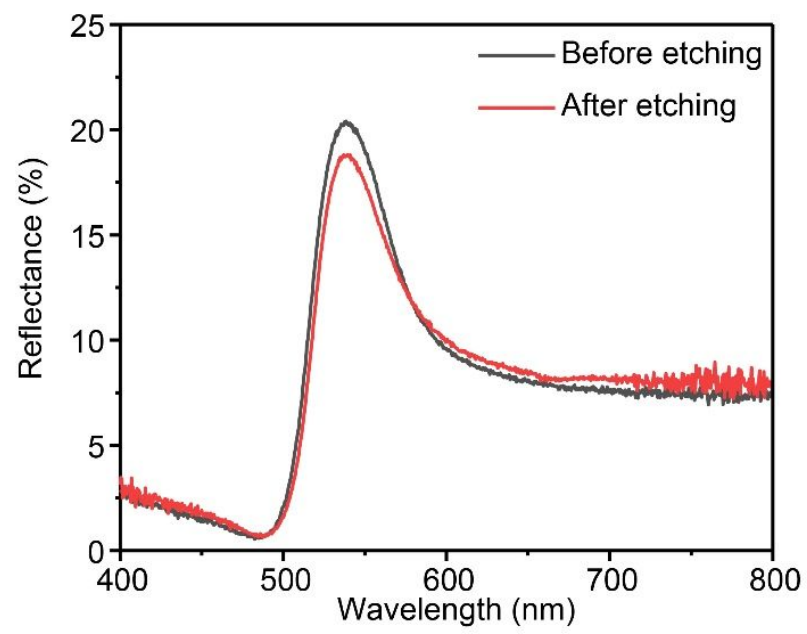

Figure S2. Reflection spectra of the PC films assembled by $\mathrm{ZnS}$ nanospheres and polymer, before and after etched by HF solution. 


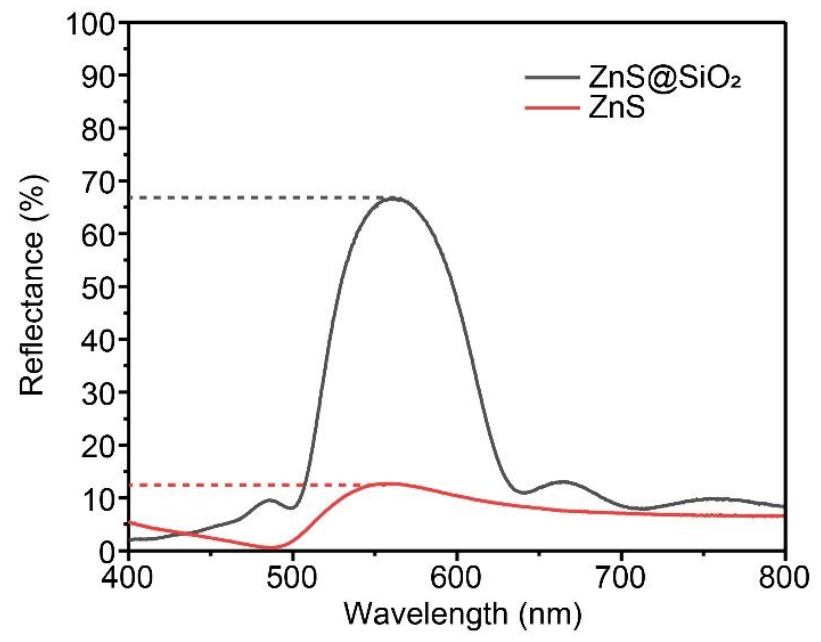

Figure S3. Reflection spectra of the PC films assembled by $\mathrm{ZnS} @ \mathrm{SiO}_{2}$ nanospheres and $\mathrm{ZnS}$ nanospheres, respectively. 

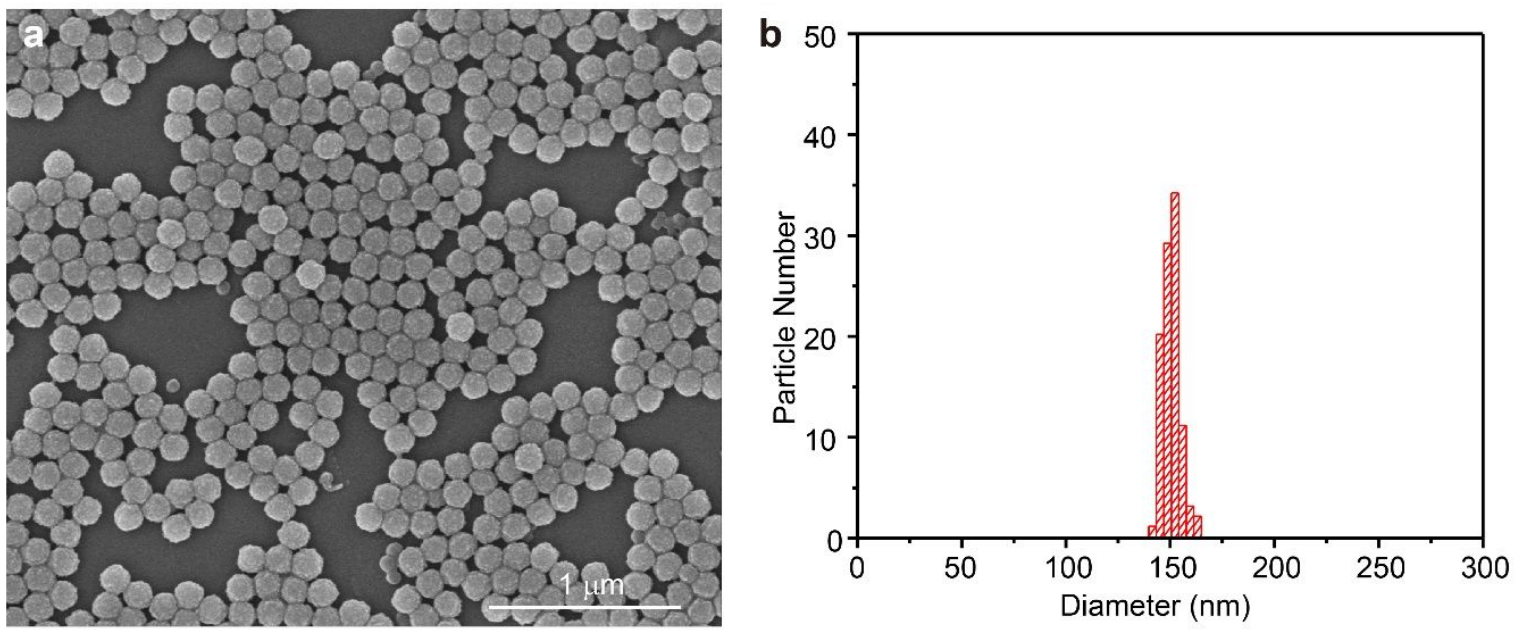

Figure S4. (a) SEM image of the synthetic monodisperse $\mathrm{ZnS}$ nanospheres with a diameter of 150 nm. (b) Size distribution of the monodisperse $\mathrm{ZnS}$ nanospheres. 

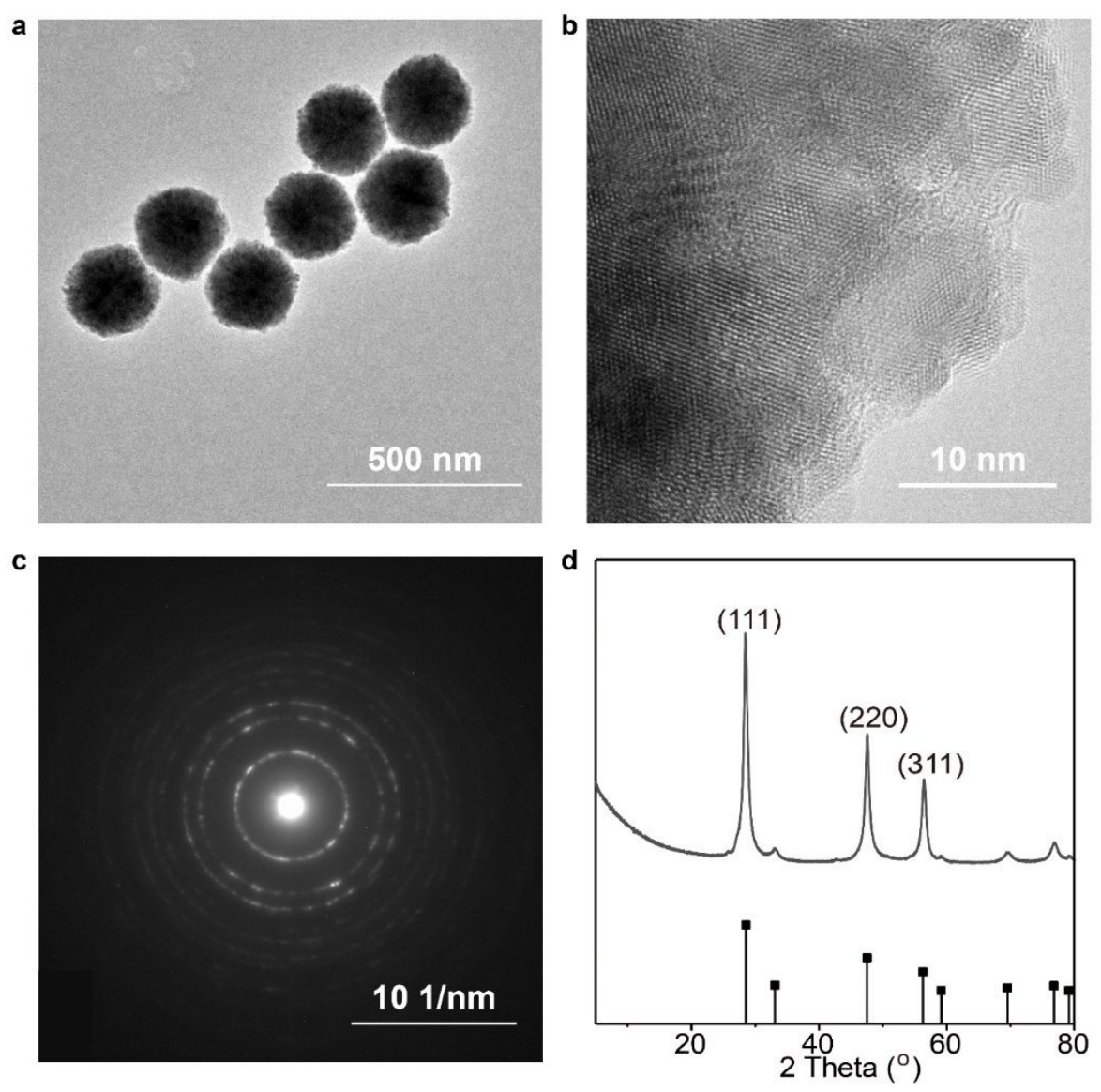

Figure S5. (a-c) TEM, HR-TEM, and SAED images image with different magnification times of the prepared $\mathrm{ZnS}$ nanospheres. d) XRD patterns of the $\mathrm{ZnS}$ nanospheres, the black vertical line is the standard card of pure cubic ZnS (JCPDS card number: 05-0566). 

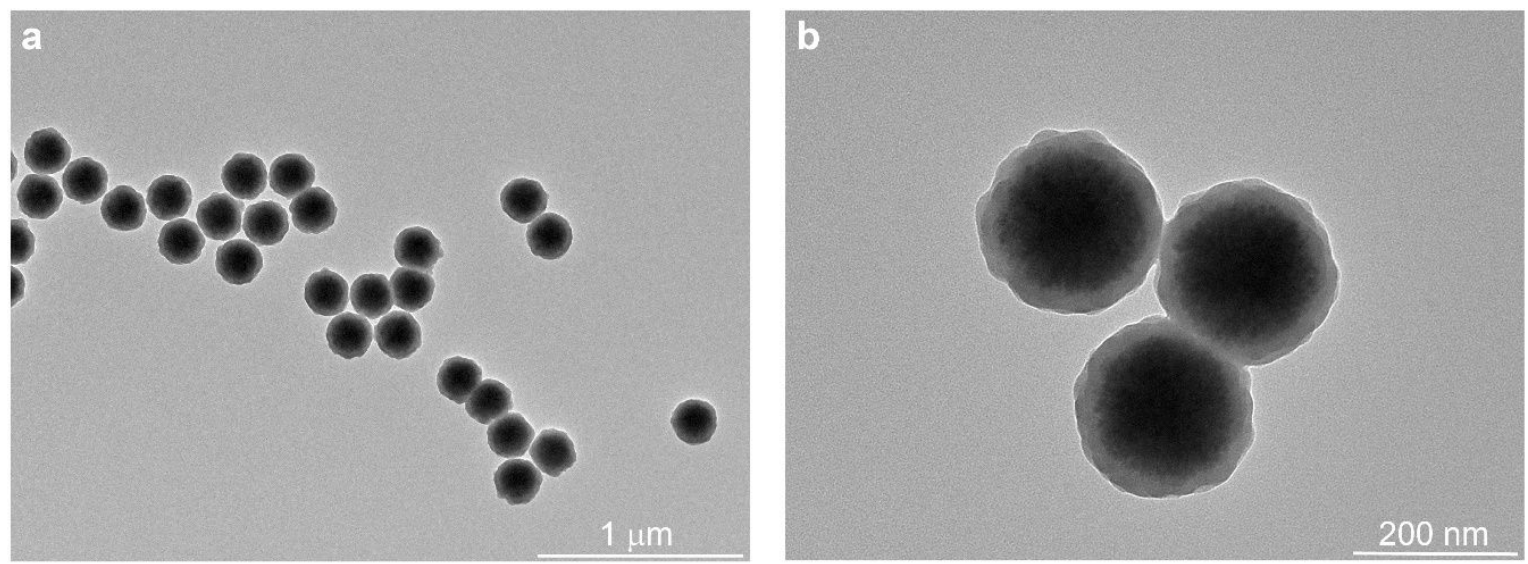

Figure S6. (a-b) TEM images image of the prepared $\mathrm{ZnS} @ \mathrm{SiO} 2$ nanospheres at different magnification times. 
a

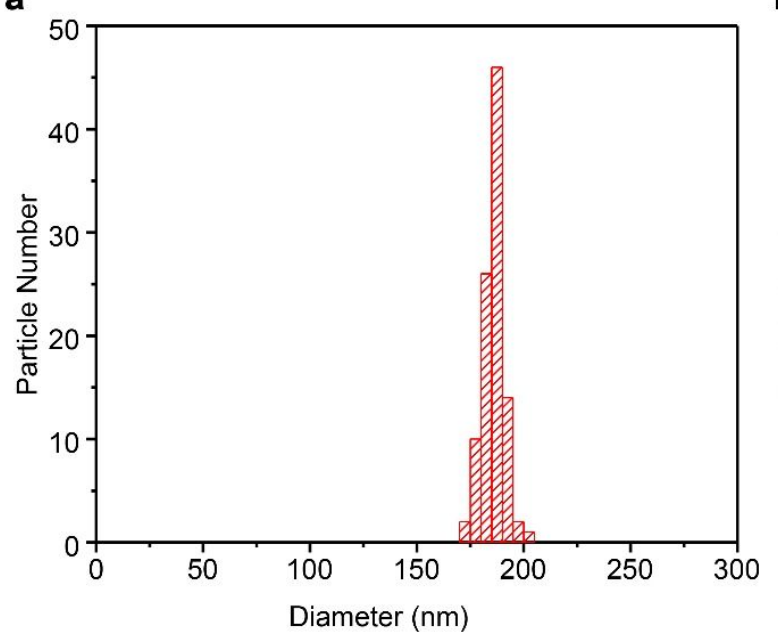

b

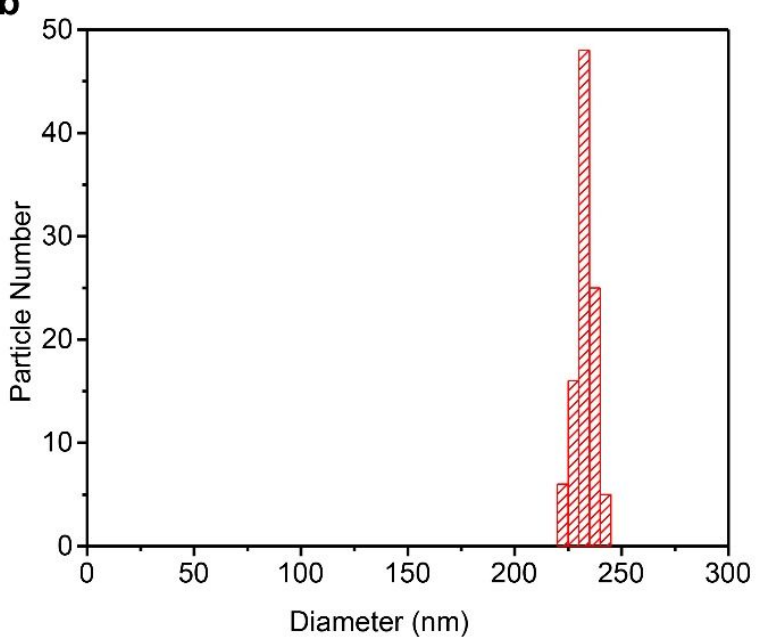

Figure S7. (a-b) Size distribution of the $\mathrm{ZnS} @ \mathrm{SiO}_{2}$ nanospheres with different diameters. 


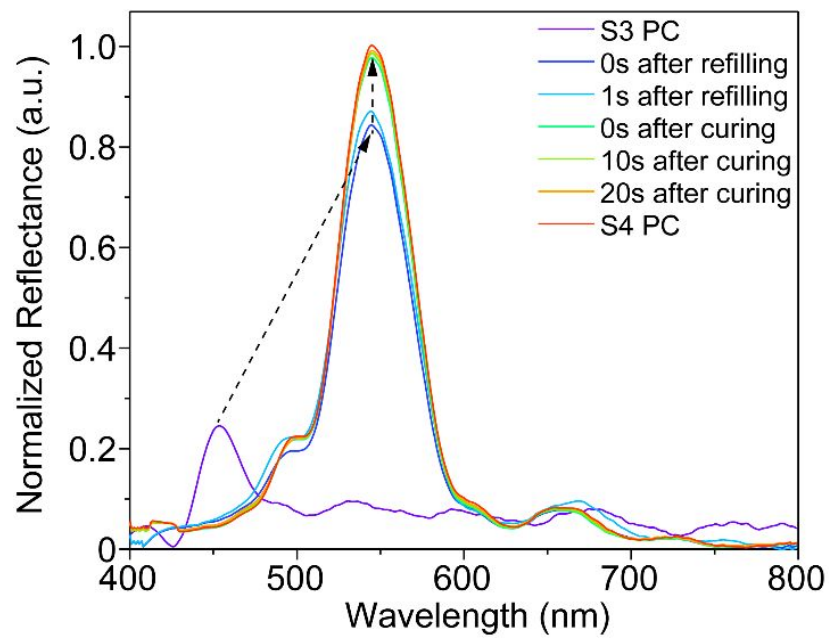

Figure S8. Reflection spectra of the PC films during the transition from S3 to S4. 
a

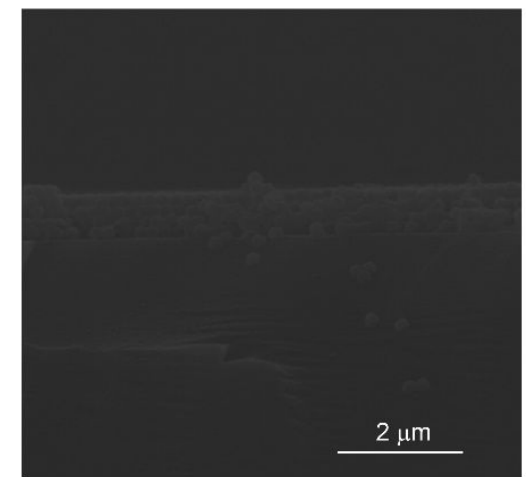

C

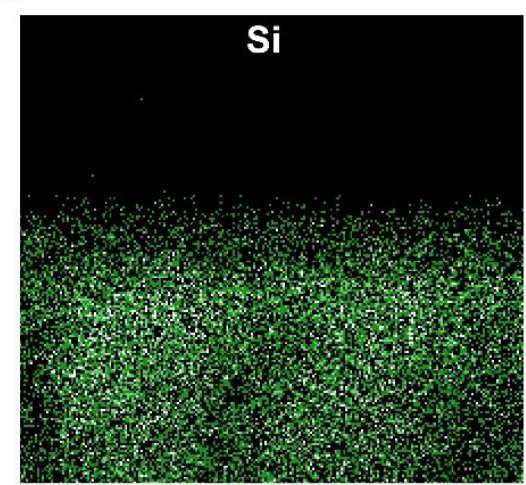

b

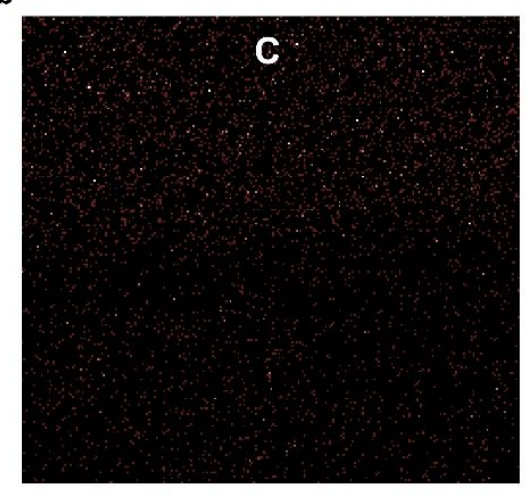

d

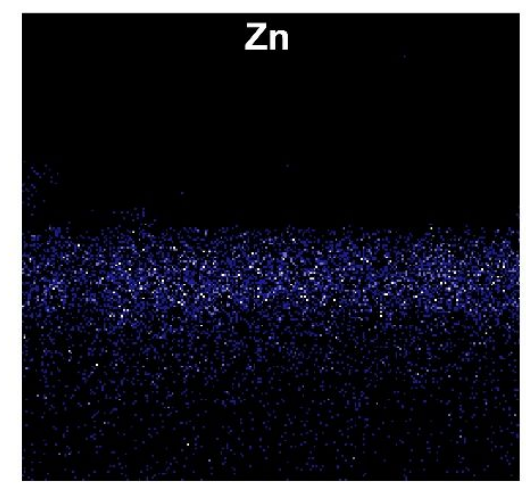

Figure S9. (a-d) SEM images and corresponding element maps of the S1 PC films: (b) carbon, (c) silicon, (d) zinc. 
a
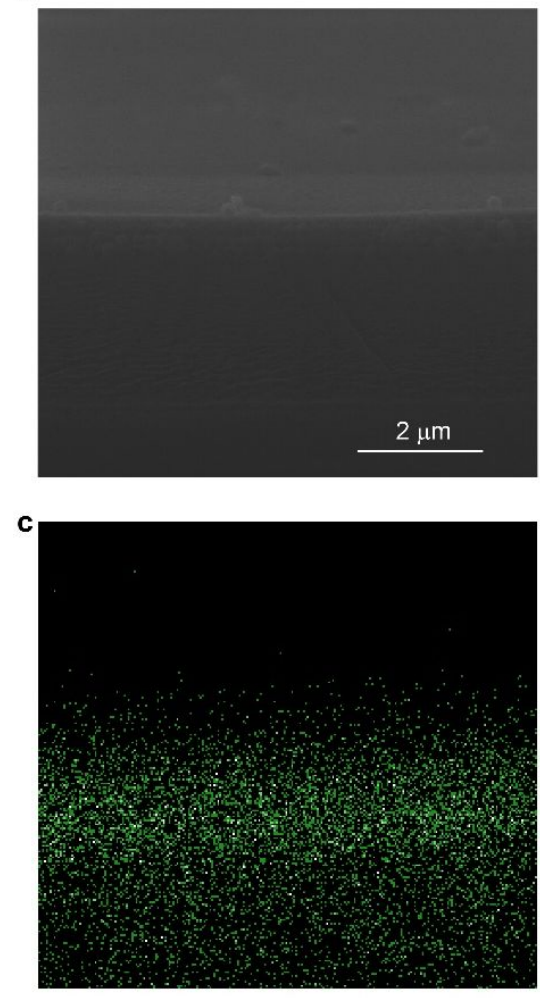

b

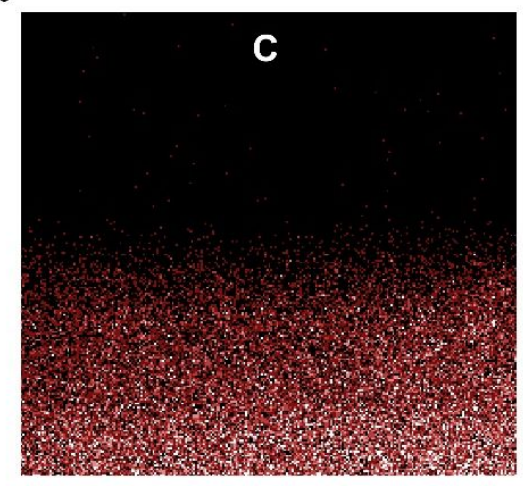

d

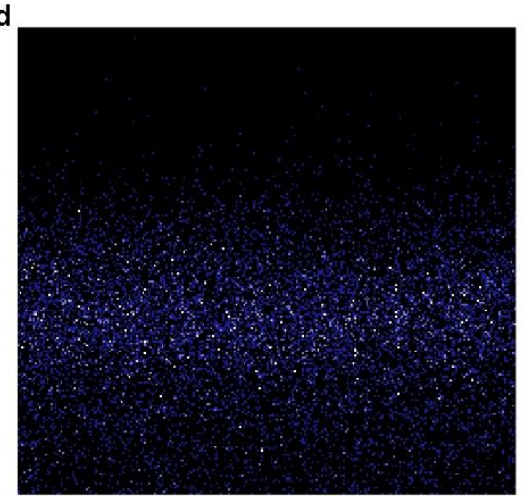

Figure S10. (a-d) SEM images and corresponding element maps of the S2 PC films: (b) carbon, (c) silicon, (d) zinc. 
a

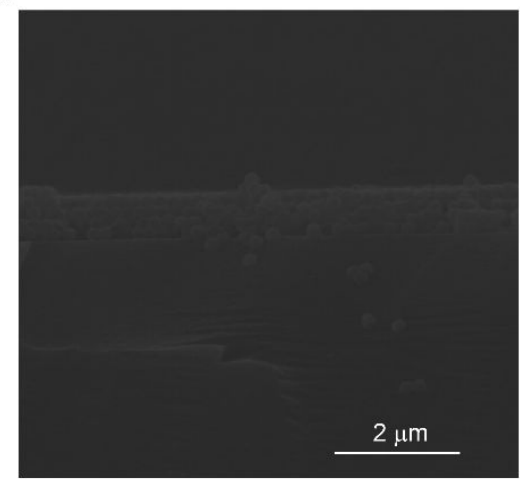

C

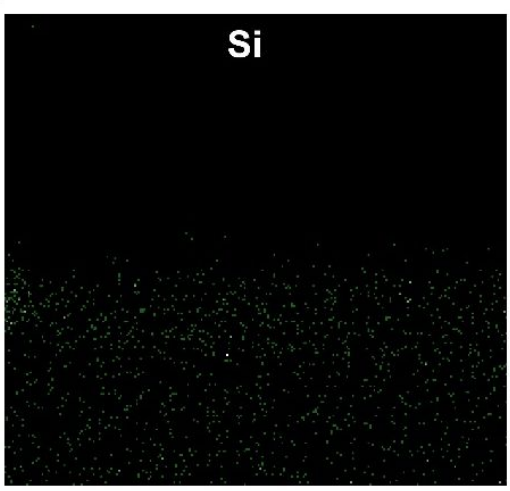

b

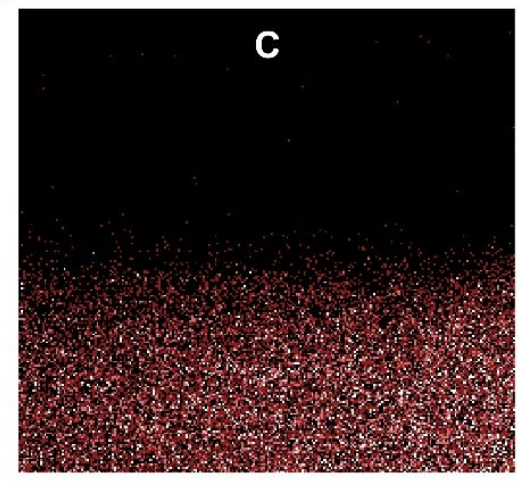

d

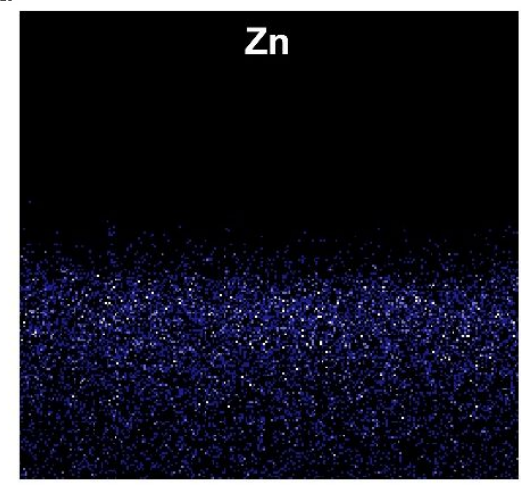

Figure S11. (a-d) SEM images and corresponding element maps of the S3 PC films: (b) carbon, (c) silicon, (d) zinc. 
a

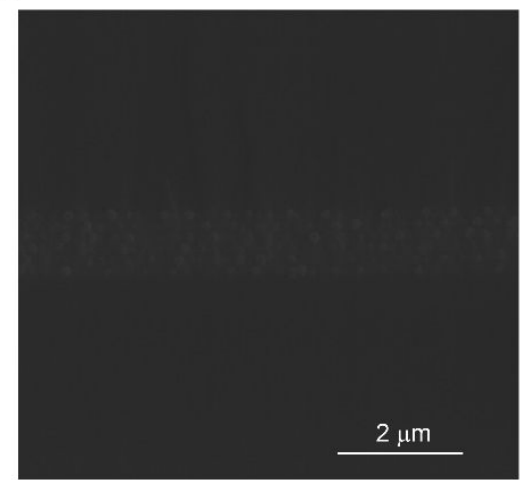

c

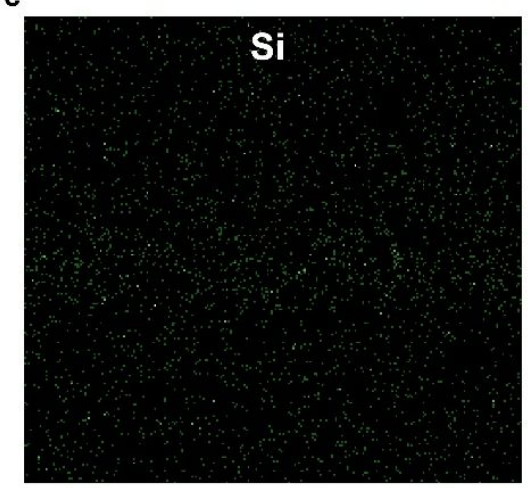

b
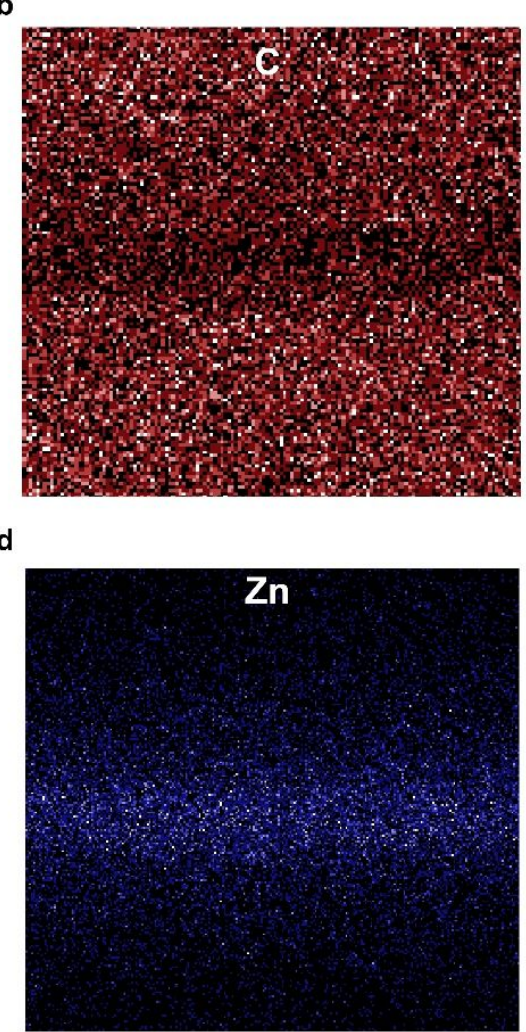

Figure S12. (a-d) SEM images and corresponding element maps of the S4 PC films: (b) carbon, (c) silicon, (d) zinc. 

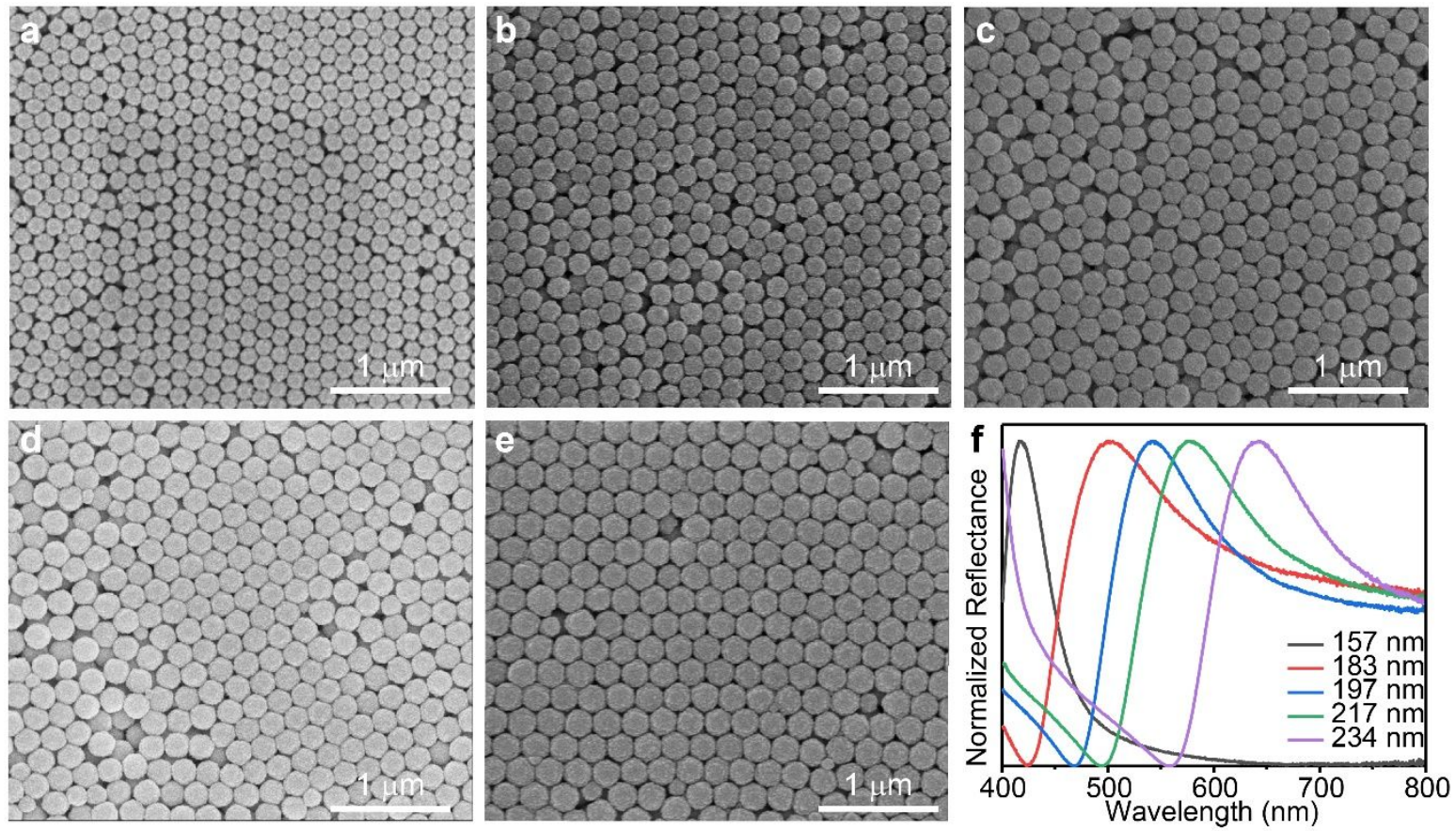

Figure S13. (a-e) SEM images of the PC films composed of $\mathrm{ZnS}$ nanospheres with different diameters: (a) $157 \mathrm{~nm}$, (b) $183 \mathrm{~nm}$, (c) $197 \mathrm{~nm}$, (d) $217 \mathrm{~nm}$, and (e) $234 \mathrm{~nm}$. f) Reflection spectra of the PC films constructed by $\mathrm{ZnS}$ nanospheres with different diameters (the measured angle is $\left.10^{\circ}\right)$ 

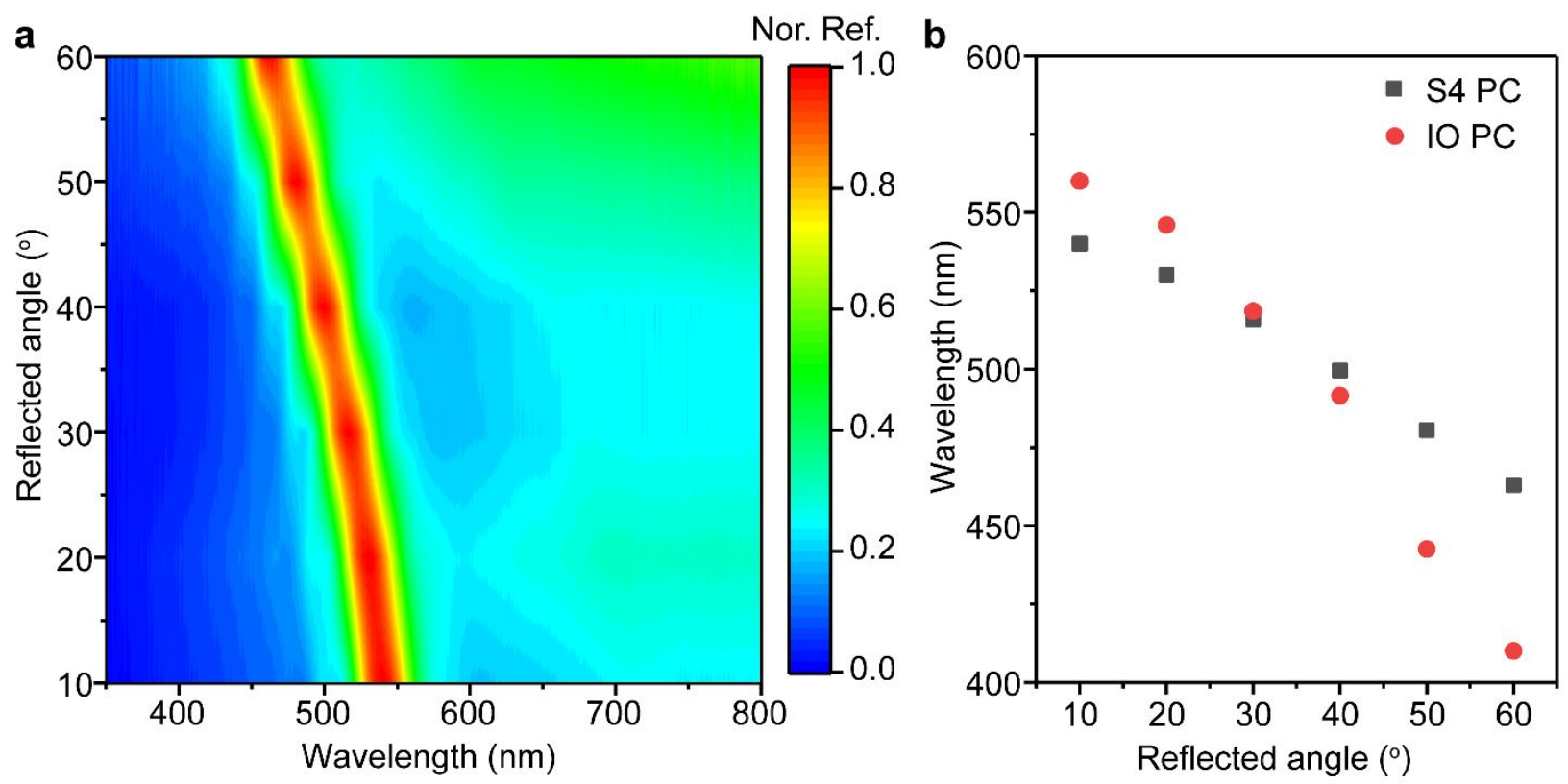

Figure S14. (a) Color-filled contour map of reflectance spectra of the S4 PC with different reflected angles. (b) Relationship of the reflected angle in the measurements and the wavelength of reflection peak of the S4 PC and IO PC, respectively. 

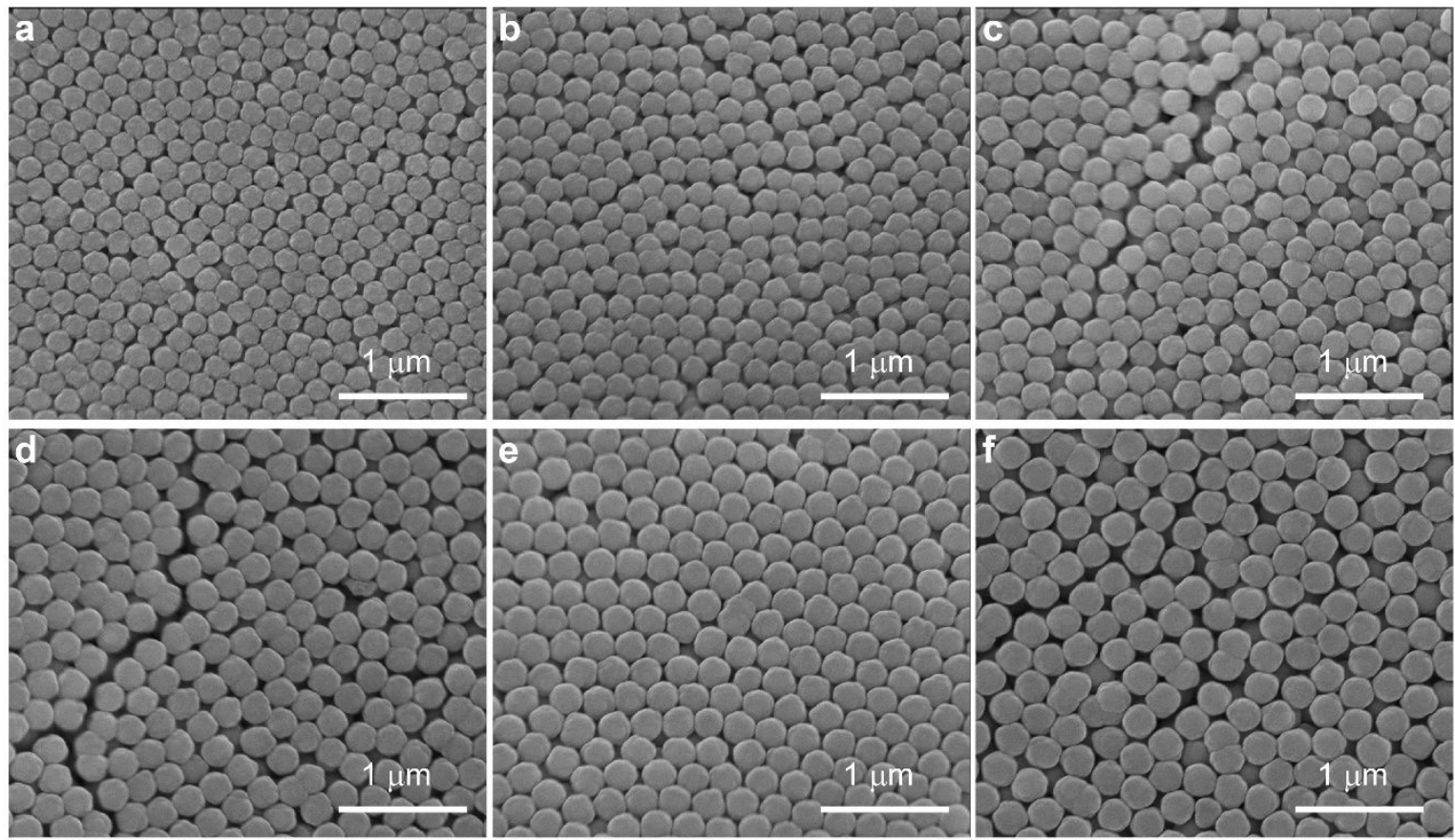

Figure S15. (a-f) SEM images of the $\mathrm{ZnS} @ \mathrm{SiO}_{2}$ nanospheres with different silica thicknesses. 

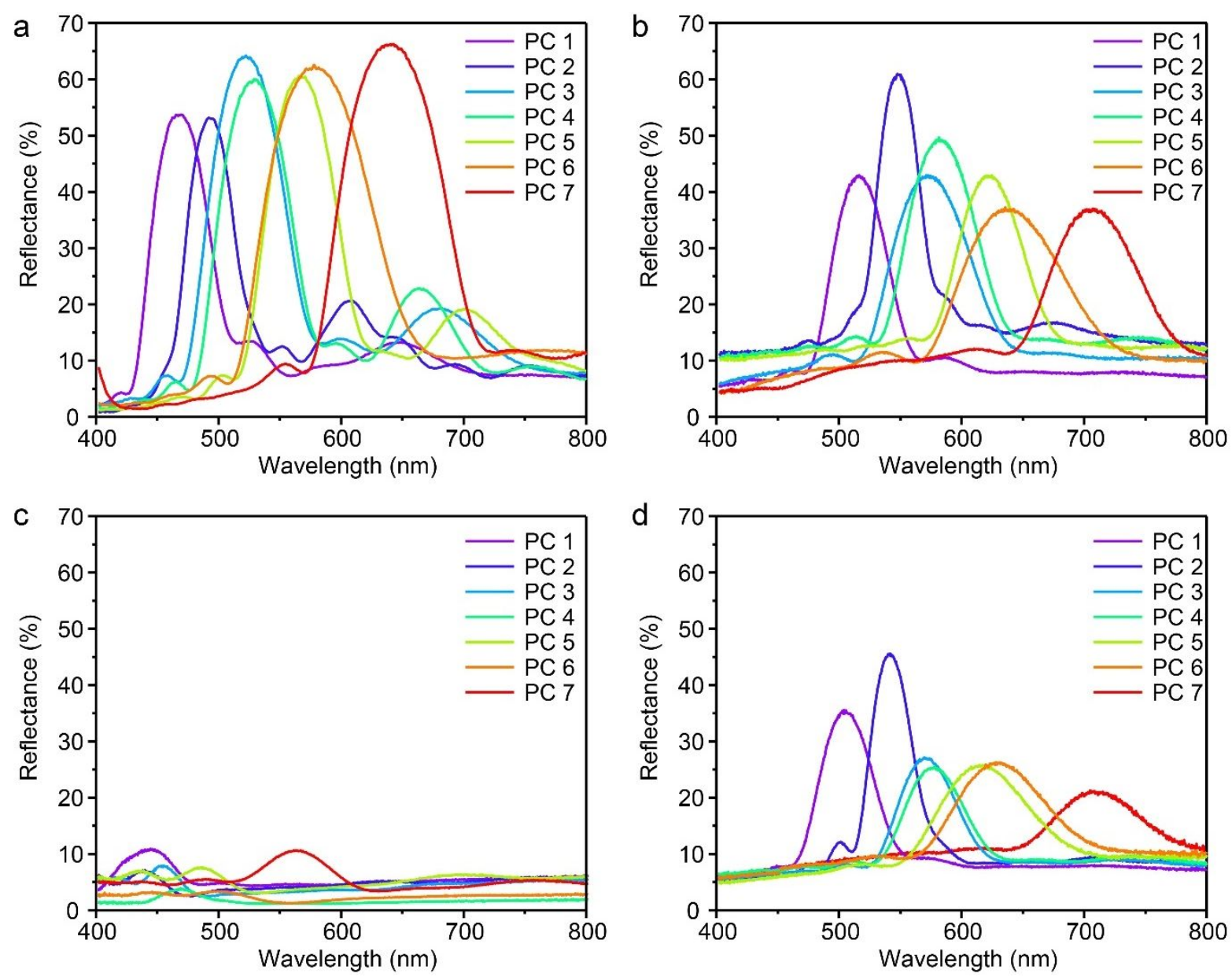

Figure S16. (a-d) Reflection spectra of the PC films composed of $\mathrm{ZnS} @ \mathrm{SiO}_{2}$ nanospheres with different diameters at different stages during the fabrication process of the NCP PC films: (a) state 1, (b) state 2 , (c) state 3 , (d) state 4 . 


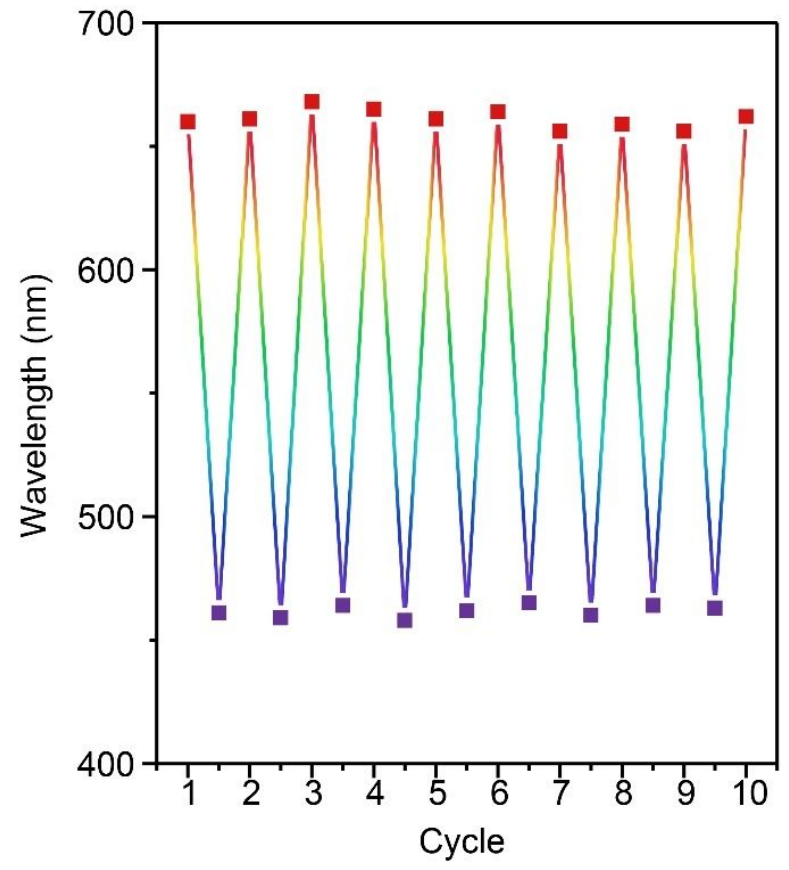

Figure S17. Reflection cycles of the thermoresponsive NCP PC film when placed alternately in two environments at $30^{\circ} \mathrm{C}$ and $55^{\circ} \mathrm{C}$. 

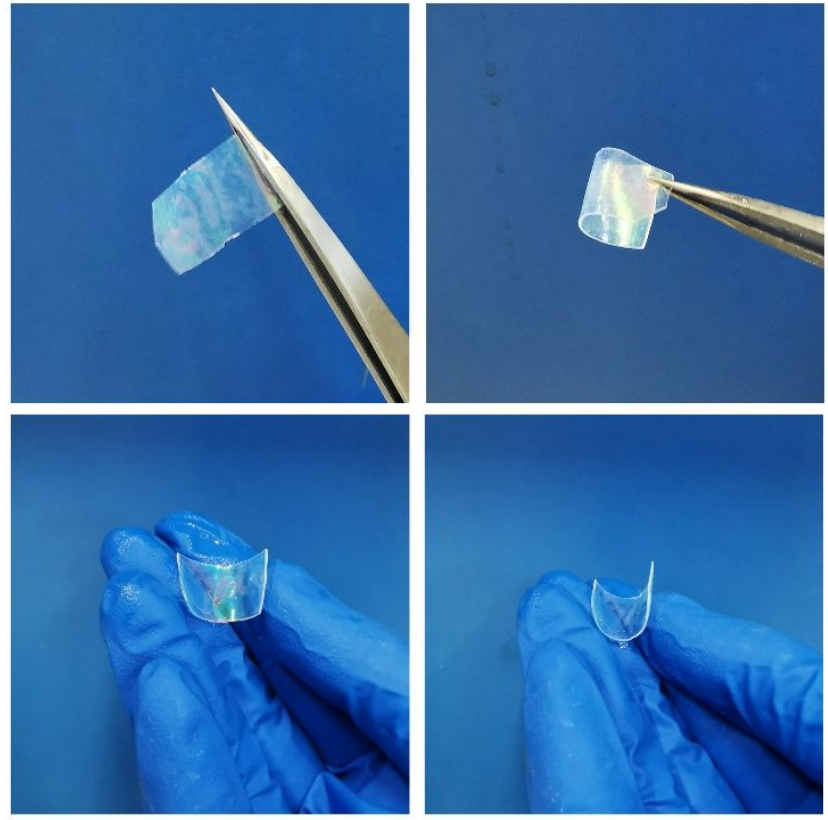

Figure S18. Digital images of the NCP PC film composed of ZnS nanospheres and PNIPAmbased hydrogel. 


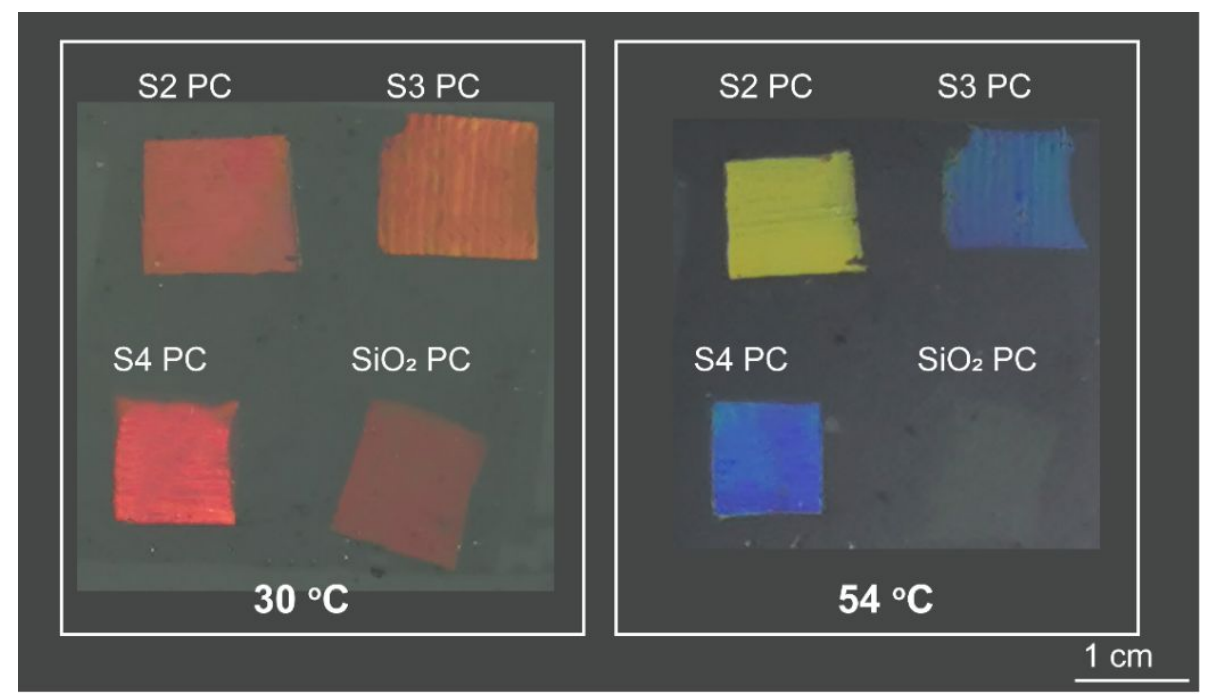

Figure S19. Digital photographs of different PC films under different temperatures. 


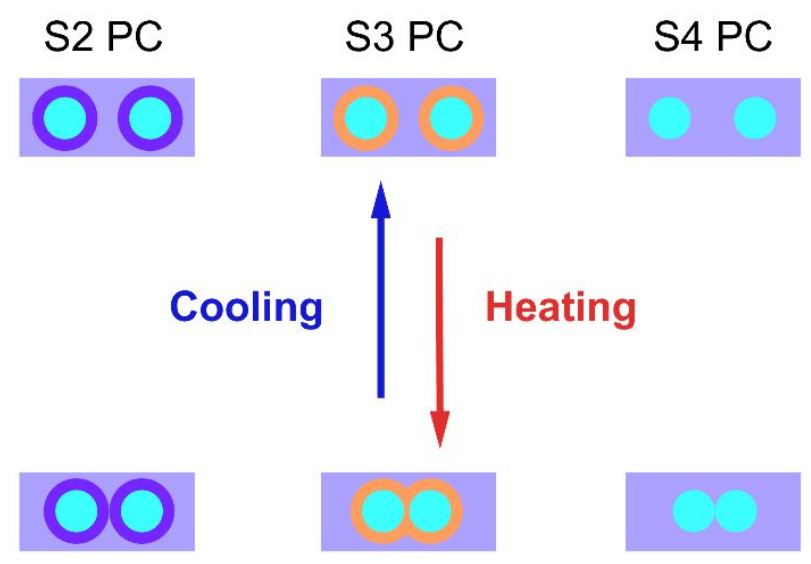

Figure S20. Schematic illustration of the dynamic range of interparticle distances in S2, S3, and S4 PC structures. 


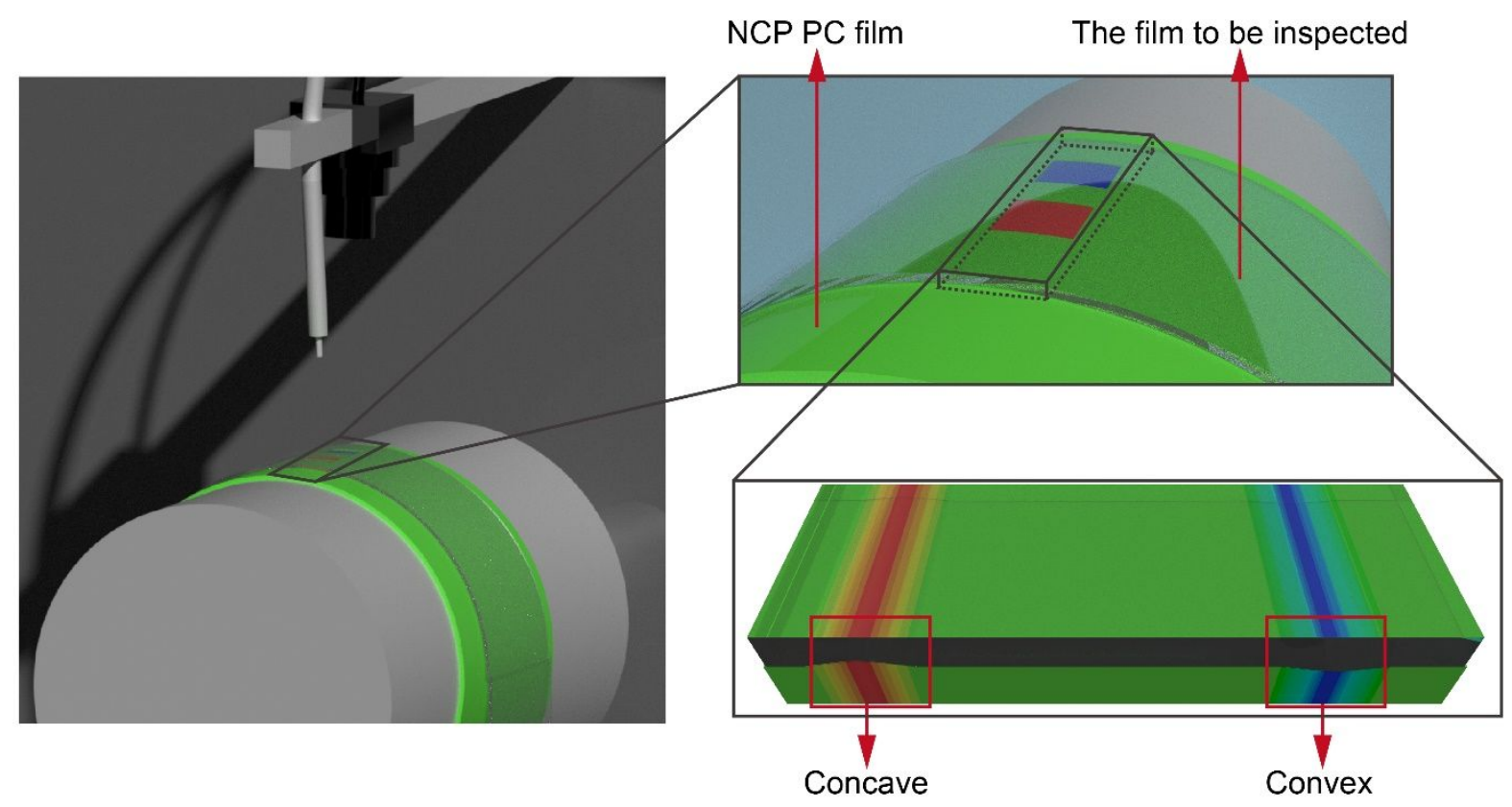

Figure S21. Schematic illustration of the roll-to-roll equipment for the flatness detection of flexible film. 